\title{
The Limbic Brain
}




\title{
The Limbic Brain
}

\section{Andrew Lautin, M.D.}

\author{
Clinical Associate Professof of Psychiatry \\ Department of Psychiatry \\ New York University School of Medicine \\ New York, New York \\ and \\ Attending Psychiatrist \\ Department of Veterans Affairs Medical Center \\ Manhattan Campus \\ New York, New York
}

\section{Kluwer Academic Publishers}

New York, Boston, Dordrecht, London, Moscow 
eBook ISBN: $\quad 0-306-46811-5$

Print ISBN: $\quad$ 0-306-46086-6

(C2002 Kluwer Academic Publishers

New York, Boston, Dordrecht, London, Moscow

Print C2001 Kluwer Academic/Plenum Publishers

New York

All rights reserved

No part of this eBook may be reproduced or transmitted in any form or by any means, electronic, mechanical, recording, or otherwise, without written consent from the Publisher

Created in the United States of America

Visit Kluwer Online at:

http://kluweronline.com

and Kluwer's eBookstore at:

http://ebooks.kluweronline.com 


\section{To \\ Arthur and Fredda Terry, Sam, and Olivia}

"As Karl Pribram has said, 'Giveme the freedom of seven neurons and I will take you from any one place to any other place in the nervous system."

—Lacey

"It is now known that the march of neural processing through the neocortex typically involves a sequence of association areas, and that a destination on the march seems invariably to be the hippocampus or the amygdala, or both."

-W. Nauta

We shall not cease from exploration

And the end of all our exploring

Will be to arrive where we started

And know the place for the first time.

T.S. Eliot, Four Quartets 


\section{ACKNOWLEDGMENTS}

A man will turn over half a library to make one book

-Samuel Johnson

Acknowledgments can start anywhere. But one has to choose a place and time. Following Samuel Johnson's suggestion I will start with an expression of thanks to Erik Helman, and Tom Waugh, librarians at the Manhattan Veterans Administration Medical Center, New York, for their unceasing efforts in finding hard to acquire articles and books. From the department of Neurology at the New York University, I would like to express my gratitude to Drs. Edwin Kolodny, Herman Weinreb, and Richard Hanson, for providing the chance to present in lecture form the material that would subsequently become the chapters of this book. I am also indebted to Drs. Derby, and Cravioto for their open invitation to attend their distinctive, and engaging, weekly brain cutting lecture series, held in the basement pathology "theater" of the VA. Closest to home I would also like to thank Drs. Sam Gershon, John Rotrosen, Bert Angrist, and Adam Wolkin, from the department of psychiatry. Martin Roth (University of Cambridge psychiatrist) wrote that all psychiatric diagnoses are hypotheses. Sam Gershon's unit at Bellvieu followed this principal providing the open, and supportive environment that would allow my early interests in neuroanatomy to develop.

I also express my gratitude to Michael Hennelly and Jennifer Stevens at Plenum Publishers. Michael a senior editor at Plenum afforded a wisdom, and forbearance that enabled an inexperienced author to "almost" get the manuscript right. Ms. Stevens, served in an admirable capacity to help in the completion of this book. 


\section{CONTENTS}

\section{Chapter 1}

Broca's Lobe ....................................................................................

Hemispheric Domains: Distinctive Features ...................................... 1

Limbic Lobe: Distinctive Features - Broca's Proposal ......................... 5

Distinctive Features of Limbic Lobe Anatomy:

A Closer Examination .....................................................................

Limbic Fissure ................................................................................... 7

Limbic Convolution ..................................................................... 8

An Exploration of the Hippocampal Region/Cortical Epithelial

Border (Cortical Limbus) in the Human Temporal Lobe ............ $\quad 14$

From The Rhinal/Collateral Sulcus to Hippocampal Sulcus to

Fimbriodentate Sulcus....................................................................... 17

Collateral Sulcus to The Entrance of the Hippocampal Sulcus:

The Parahippocampal Sectors (The Mesocortical Sectors)

A Survey Along the Entrance to the Hippocampal Sulcus:

Transition From Presubiculum (Mesocortex) to Subiculum

(Allocortex). Transition from TwoTiered Mesocortex to a

Single Tiered Allocortex

A Survey Along The Obliterated Hippocampal Sucus:

The Hippocampus (Hippocampus Proper) Ammons Horn

Visualizing (Unobliterating) The Hippocampal Sulcus: Sectors

CA1-CA3 on the Lateral Side of the Hippocampal Sulcus ........... 22

A Brief Review of the Territory Covered ......................................... 23

Fundal Area of Obliterated Hippocampal Sulcus:The Zone of

Transition from CA3 to the Dentate Gyrus

The CA3 to Dentate Gyrus Transition (Along the Surface of the

Dentate Gyrus); and, the CA4 to Dentate Gyrus Transition

(Into the Hilus of the Dentate Gyrus) ........................................ 23

Dentate Gyrus to Fimbriodentate Sulcus ......................................... 24

Hippocampal Terminology .................................................................... 24

Cortical Terminology ...................................................................... 27

Terminology of the Iso, Meso-, and Allocortex:

Issues Related to Evolutionary Age .......................................... 28

Pallial Terminology: Medial, Dorsal, and Lateral Pallial

Districts ..................................................................................... 28

The Medial Pallium .......................................................................... 33 
The Dorsal Pallium ....................................................................... 33

The Lateral Pallium ........................................................................ 33

Medial, Dorsal, and Lateral Pallial Districts:

Brief Introduction to Hodology ………........................................ 34

Medial Pallium ............................................................................. 34

Dorsal Pallium ............................................................................. 35

Cortical/Hippocampal Terminology:Implications for the Growth Ring Hypotheses ....................................................... 37

The Hippocampal Region: a survey of its Course Throughout the Hemisphere.................................................................................... 38

White Matter Side of the Hippocampal Region: Alveus to the Great Midline Hemispheric Commissures .................................... 42

Alveus to Fimbria ……................................................................. 42

Great Commissures of the Midline: (Fimbria-Fronix, Corpus

Callosum, Anterior Commissure) …………………......................... 43

Development of the Corpus Callosum ................................................. 44

Extended Definition of the Hippocampal Formation ........................ 47

Broca's Limbic Lobe: An Example of the Search for

Hemispheric Archetype .................................................................. 47

A Tabular View of the Limbic Brain Concept .................................... 48

Nauta's Septo-Hypothalamic Continuum: Introduction to

a Linear Limbic Icongraphy ........................................................ $\quad 50$

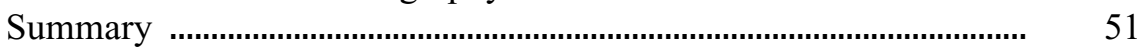

References ................................................................................. 52

\section{Chapter 2}

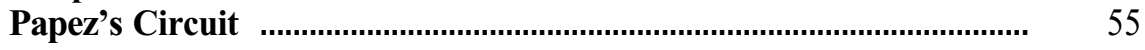

Introduction to Papez's Circuit .............................................................. 55

Brain Stimulation and Transection ..................................................... 58

Brain Transection: Pseudaffective Reflex State .............................. $\quad 58$

Brain Transection: Sham Rage ....................................................... 59

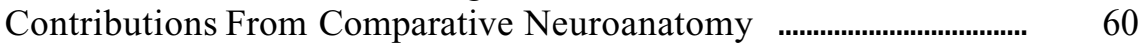

Summary to Previous Sections ............................................................ 63

Anatomic Mediation of Emotions (Awareness): Basic

Components

The Cingulate: Cortical Mediator of Emotional

Experience ...................................................................................... 64

Papez's Circuit ................................................................................... $\quad 66$

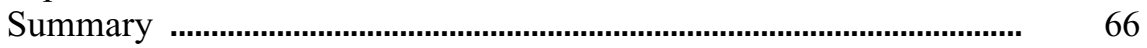


Towards a Limbic System ............................................................... 67

References .................................................................................. 67

\section{Chapter 3}

MacLean's Limbic System .................................................................

Introduction to MacLean's Contribution .............................................. 69

The Dissemination of Papez's Proposed Mechanism of Emotion ..................................................................................... $\quad 70$

From Papez's Circuit to MacLean's Limbic System ........................... 73

Contributions from Neuronography to the Development of the Limbic System Concept ...................................................... 73

The Contribution of Clinical and EEG Findings to MacLean's Limbic System Concept ............................................................ 74

Summary

An Ambitious Synthesis:The Limbic Brain in an Evolutionary

Biologic Context ....................................................................... $\quad 78$

Phyletic Considerations .................................................................... 80

An Introduction to the Triune Brain Concept ............................... 81

Comparative Behavioral Profiles Analysis: Contrasting Reptilian

(R-Complex) and Mammalian (Paleomammalian/Limbic)

Mediated Behavior

Placententation, Viviparity, Mammary Glands and Increased

Maternal Care .............................................................................. 86

Audiovocal Communication - The Development Of Middle

Ear Bones

Neoteny: Enhance Maternal Care: Our Highly Neotenous

Brain

Controversy Surrounding MacLean's Triune Brain Construct ...............

Limbic Components Evident Throughout the

Vertebrate Series

Newer Insights into Limbic Brain Evolution Provide Limited

Support for MacLean's Theory? ...................................................... $\quad 92$

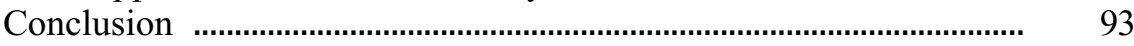

Appendix ......................................................................................... 93

Entopallium; Mesopallium; Ectopallium: Three Layers of the

Hemisphere ................................................................................. 94

Telencephalon Impar, Semipar, and Totopar ..................................... 95

Telencephalon Impar ................................................................. 95

Semipar \& Totopar ........................................................................ 97

Bibliography ........................................................................................ 97 


\section{Chapter 4}

Nauta's Limbic Midbrain .......................................................................... 99

Nauta's Limbic Midbrain: Introduction ............................................... 99

The Concept of the Limbic Midbrain Area ………................................ 100

Nauta's Limbic Midbrain: Methods; Finding; Pathways ..................... 103

The Medial Forebrain Bundle (MFB) ........................................... 105

The Stria Medullaris (SM) and the Fasciculus Retroflexus (FR) .... 108

Mammillotegmental Tract ............................................................... 108

Summary to Section ......................................................................... 108

Interconnections of Limbic Midbrain Circuit to Spinal and Frontal

(Neocortical) Districts ................................................................ 109

The Use of Newer Methods Demonstrate Direct Afferentiation of Limbic Cortex from the Paramedian Midbrain: Fluorescence

Microscopic Mapping of the Ascending Monoamine System ........

Technical Considerations ................................................................. 112

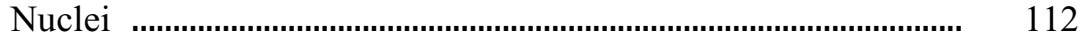

Ascending Tracts ......................................................................... 114

An Extended Limbic System: Neurobehavioral Implications ............... 116

Conclusion .......................................................................................... 118

References ........................................................................................... 119

\section{Chapter 5}

Heimer's Limbic Stricatum ............................................................... 121

Subcortical Projections of the Allocortex: Anatomical Implications for the Limbic System Concept .................................................. 121

Allocortical to Ventral Striatal Projections ...................................... 122

Ventral Striatal to Ventral Pallidal Projections (The 1st description of the v.pallidum)

Similarities Between The Subcortical Projection Fields of the Allocortex and Neocortex

Limbic Striatal Integration - a Model for the Functional Interface

Between Limbic and Motor Systems

The Basal Forebrain in Limbic Motor Integration ............................. 131

Wiring Diagram - The Model ........................................................ 132

Conclusion

References

Index 


\section{CHAPTER SUMMARIES}

The book, which is divided into five chapters, overviews basic concepts and principles in our understanding of limbic brain anatomy dating to Broca's proposal in 1878. In the first four chapters, the limbic brain is examined as a somewhat independent hemispheric domain. Broca's great limbic lobe, Papez's circuit, MacLean's limbic system, and Nauta's limbic midbrain form the conceptual core of chapters $1-4$, respectively. The inextricable linkage of limbic and neighboring districts, implicitly acknowledged in the first four chapters, receives particular emphasis in chapter 5, a chapter, which addresses the conceptual issues put forth by Heimer and Wilson in their seminal publication on the ventral striatum.

\section{CHAPTER 1}

This chapter is intended to achieve four goals. An overview of several classical $\left(19^{\text {th }}\right.$ century $)$ descriptions related to the limbic brain concept. These include Rolando's processo cristato, Gerdy's circonvolution annulaire, and of course, Broca's great limbic lobe. A survey of the hippocampal formation and cortical limbic border in mid temporal section (at the body of the hippocampus) using recent illustrations, and terminology the chapter will provide an examination of how Broca's lobe relates to later conceptual developments in the fields of cytoarchitectonics, and comparative neuroanatomy will also be provided.

\section{CHAPTER 2}

In 1937, James Papez would see in the ensemble of the hypothalamus, cingulate gyrus, and their connections an anatomic theory of emotion. Francis Schiller remarked that Papez's idea would go on to create something of a stir. Kenneth Livingston referred to Papez's proposal as the most dramatic shift in emphasis in our view of limbic structures since the time of Broca. Seeming to capture the essential element in Papez's contribution, Paul Yakovlev wrote

"In his now classic article of 1937, he [Papez] humbly proposed, and at some calculated risk, that 'emotions' have an anatomical 'mechanism,' and he marshaled evidence for the location of the mechanism in the brain. This empirical generalization contradicted the notion widely accepted in 
his time, that experience so exquisitely 'mental,' as are 'emotions are a preserve of psychology and that a generalization proposing an anatomical mechanism was a delusion."

This chapter examines the conceptual developments leading Papez to his proposal of an anatomic theory of emotion. Three conceptual underpinnings of Papez's idea are traced.

The results of transection experiments (Sherrington, Cannon, and Bard) conducted in the early $20^{\text {th }}$ century which pointed to the importance of a forebrain mediator of emotional expression.

The emerging neuroanatomic, and neurobehavioral opinion in the first decades of this century, which maintained a primacy for medially arrayed hemispheric structures (i.e. the hippocampus, amygdala, and neighboring rostral diencephalic/hypothalamic loci) in the mediation of neurovisceral, endocrine, autonomic nervous system, and interoceptive processes. This view also held that the laterally displayed hemispheric wall structures served primarily somatic and exteroceptive processes.

The assumption (based on clinical, and theoretical grounds, and analogizing emotional awareness to other known sensory processes) that emotional consciousness required a cortical (not subcortical or peripheral) mediator. Papez would chose the mesocortex of the cingulate gyrus as the principal (but not the only) receptive area for the experience of emotion.

Cohering these areas, Papez put forth his idea of an anatomical circuit, which, in his own words, would serve as "a harmonious mechanism" mediating emotion. Papez's proposal was an early well principled anatomically determined theory of emotion. Emotional awareness/consciousness received no special privilege in Papez's theory, indeed the emotional sense would be brought into the same anatomic theater of explanation as the general somesthetic and special senses: i.e. similar to pain, temperature, and vision, emotional experience was accorded a thalamic nucleus and thalamocortical cortical relay. Coleridge's lines are evocative of Papez's grand conjecture.

"I have not only completely extricated the notion of time, and space... but I trust that ; am about to do more-namely, that ; shall be able to evolve all the five senses, that is to deduce them from one sense \& to state their growth, \& the causes of their difference $-\&$ in the evolvement to solve the process of Life and Consciousness.

SAMUEL COLRIDGE (1801).

Chapter two is devoted to a review of Papez's rather extraordinary rendering of core limbic function. 


\section{CHAPTER 3}

From the early nineteenth century to Papez's proposal put forth in 1937, a favored view of higher mammalian limbic anatomy was that of a circumannular convolution, hugging the mid sagittal plane, and limned (bordered) by the limbic fissure. However within two decades of Papez's paper the limbic brain concept would be transformed to include a consortium of neural structures extending rostral to the limbic fissure, yet intimately connected with Papez's core circuit elements. Brought into the limbic ambit were the orbitofrontal cortex, also perihippocampal districts. No longer constrained to the midsagittal plain the rather exuberantly conceived limbic coalition would be called a limbic system. One of the principal figures who brought about this transformation was Paul MacLean. MacLean also engaged in an enterprising endeavor to place the limbic system of mammals in a broadly conceived evolutionary context. In this chapter, we examine the contribution of MacLean to limbic brain neuroanatomy and function.

Chapter 3 also includes an appendix addressing the work on limbic brain anatomy of Paul Yakovlev (1894-1983). Although Yakovlev's language and approach has been characterized as unusual - indeed Herrick, commenting on Yakovlev's seminal publication on the limbic brain said he agreed with its argument but "would have put it differently" - Yakovlev's paper on limbic brain anatomy stands alongside MacLean's and Papez, it is one paper from this famous limbic trilogy. We hope the appendix to this chapter conveys a sense of Yakovlev's contribution to the concept of the limbic brain.

\section{CHAPTER 4}

If a general trend can be said to characterize neuroanatomic research on the limbic brain at mid century then surely it was the extension of anatomic borders of limbic system to even further rostral and caudal domains of the neuraxis. Spearheaded by Walle Nauta, the limbic consortium would expand further to embrace more firmly both neocortical and midbrain structures. In this chapter we explore Nauta's concept of the limbic midbrain and examine his views on prefrontal to basal hemispheric limbic connections.

\section{CHAPTER 5}

This chapter is centered on an examination of the publication of Heimer and Wilson. This paper helped to better establish the nature of 
the anatomical interface of limbic and striatal domains. Referred to by McGeer as the twin galaxies of the inner universe, a discussion of the linkage of striatal and limbic anatomic districts also serves to de-emphasize the concept of an independent limbic brain domain, and ends our introduction to limbic anatomy. Interestingly, Heimer and Wilson, make the point that a strict interpretation of then extant limbic anatomical canons would act to forestall broad acceptance of limbic striatal integration in the first half of this century. In this chapter, avoiding this potential pitfall, we employ the basic principles of limbic brain anatomy reviewed in the earlier chapters to better appreciate the nature of Heimer and Wilson's findings. Chapter 5 concludes with a brief examination of limbic striatal integration, as put forth by Gordon Mogenson in his oft-referenced paper on limbic motor integration.

\section{REFERENCES}

1) Broca, P. (1878). Anatomie comparee des circonvolutions cerebrales. Le grand lobe limbique et la scissure limbique dans la serie des mammiferes, Rev. Anthropol. I, Ser. 8, 385-498.

2) Papez, J. W. (1937). " proposed mechanism of emotion, Arch. Neurol. Psychiatry, 38, 725-743.

3) Yakovlev, P. (1972). A proposed definition of the limbic system; in Limbic System Mechanisms and Autonomic Function (C. H. Hockman ed.), Springfield Illinois, 241283.

4) MacLean, P. (1952). Some psychiatric implications of physiological studies on frontotemporal portion of limbic system (visceral brain), Electroencephalogr, Clin. Neurophysiol. 4, $407-418$

5) Yakovlev, P. (1978). Recollections of James Papez and comments on the evolution of the limbic system concept (As told to Ken Livingston), in Limbic Mechanisms (K. E. Livingston and Oleh Hornykiewicz eds.), Plenum Press, 351-354.

6) Yakovlev, P. I. (1948). Motility, behavior, and the brain. Stereodynamic organization and neural coordinates of behavior, J. Nerv. Ment Dis. 107, pp. 313-335.

7) Nauta, W. (1958). Hippocampal projections and related neural pathways to the mid-brain in the cat, Brain 81,319-340.

8) Heimer, L., and Wilson, R. D. (1975). The subcortical projections of the allocortex: Similarities in the neural associations of the hippocampus, the piriform cortex, and the neocortex, in: Proceedings of the Golgi Centennial Symposium (M. Santini, ed.), Raven Press, New York, 177-193.

9) Mogenson, G. J.,Jones, D. L., and Yim, C. Y. (1980). From motivation to action: Functional interface between the limbic system and the motor system, Progress in Neurobiology, pp. 69-97. 


\section{INTRODUCTION}

Nearly, 50 years ago, Karl Pribram in a discussion section accompanying MacLean's proposal of a limbic system, criticized the visceral or limbic brain concept as theoretically too vague and cumbersome. In a recent review of the limbic system, Swanson points to Brodal's criticism that the discovery of connections of limbic structures with virtually all parts of the nervous system render the concept of the limbic system useless, and better abandoned.Additional dissatisfaction surrounding the limbic brain concept stems from the feeling that it is historically inert (an antiquated 19thcentury construct). In our current age of neural networks, and parallel distributed process it is of little value, merely an historical curio. So why then this introduction to limbic brain anatomy? We offer several interrelated rationales behind our labors.

Recapitulation in the Service of Education: Although concepts had evolved in the second half of this century which effectively overthrew the idea of relatively isolated hemispheric districts (i.e. striatal, cortical, and limbic), parsing the hemisphere into these three districts was an important preliminary step achieved by our forebears in their efforts to understand the large scale structure of the higher mammalian cerebral hemisphere. An examination of how the limbic brain concept came to be provides an opportunity to recapitulate the process of exploration, discovery, and understanding as it relates to one of these principle hemispheric domains.

The Strange, Natural Beauty of this Neural Landscape: The neural terrain surrounding the epithelial margin of the hemisphere (Broca referred to this cortical limbic area as the threshold of the hemisphere) has engaged the interest of anatomists for the last two centuries. The following comments by four of the leading anatomists of the $20^{\text {th }}$ century underscore the attraction, and importance of this area:

"... I may say that one of the stimuli which led me to scrutinize the hippocampus and the dentate fascia was the elegant architecture shown by the cells and layers of these centres as revealed by the illustrious Golgi in his great work. In fact, the hippocampus and the dentate fascia are adorned by many features of the pure beauty of the cerebellar cortex. Their pyramidal cells, like the plants in a garden - as it were, a series of hyacinths - are lined up in hedges which describe graceful curves."

Ramon Cajalt (4, p. 415) 
Certain of the most excellent contributions in to the history of comparative neurology have dealt with the hippocampal region of mammals, and this is true whether the general relations of the parts in the different mammals are taken into consideration .... or the finer details of structure are considered...

Ariens Kappers, Huber, Crosby, 1936 (5, p. 1410)

Little wonder, all in all, that Broca decided he had found the edge of the cerebral cortex, at least in the temporal lobe, and little wonder that he initially chose to call his discovery the great lobe of the hem. Here in the temporal lobe, the edge is clearly arrayed in a way suggesting the strategy of a seamstress who finishes off the hem of a garment by folding its material several times and even placing over its end an extra piece of fabric before stitching it all together.

$$
\text { NAUTA, } 1986 \text { (6, pp. 274-275) }
$$

Therefore, as I guide the reader through the strange landscape of the hippocampal region, I remind him of the history of search and discovery here, a history that can be perused with pleasure and scholarly satisfaction." (White, Yakovlev) ...

J. ANGEVINE, 1978

A principle goal of this book is to follow in the footsteps of such intrepid explorers, journey into this alluring neural landscape; and learn the secrets of its design, and function.

The Anatomy of Consciousness - Early Theories:Throughout much of the last century, more attention was directed to the limbic neural consortium (including the rostral brain stem) in the mediation of consciousness and emotional awareness, than on structures of seemingly equal complexity, such as the cerebellum. Why is this? Several anatomic theories which have attributed a central role of limbic (or equivalently centrencephalic) anatomy to the mediation of consciousness have been put forth, a partial list includes Herrick's view (and it would seem Broca's view as well for Broca also spoke of this issue), which held that the olfactory system established motivational contexts, and directly motivated behaviors. Papez's proposed circuit elaborating emotion, MacLean's limbic system, Yakovlev's paper on the three spheres of motility and behavior, Olds and Milner's discovery of the reward systems of the brain, notably their indwelling electrodes were positioned in what they referred to as the rhinencephalon, high rates of self stimulation were recorded in the mammillothalamic tract and cingulate cortex (11, P.425). These two structures are cardinal elements in Papez's circuit. Heimer and Wilson's proposal (actually a re-conceptualiza- 
tion) of the basal forebrain and associated limbic loci as a macro-structure enabling motivation to become action, and relatedly Mogenson's celebrated paper addressing the same theme of motivation transmuted into action in the midbrain and basal forehair. An historical overview of our attempts to relate brain, consciousness and emotionality, would be incomplete without surveying such limbic centered proposals.

In Search of the Unity of Type:Broca's proposal of the great limbic lobe (a great inner ring of tissue embedded in all mammalian brains) can (and should) be seen as an elegant contribution by anatomists to reduce neuroanatomic (cerebral hemispheric) diversity to one or very few underlying architectural themes or building blocks. This grand romantic vision (the aspiration of transcendental morphology) had received its clearest voice earlier in $19^{\text {th }}$ century France with Etienne Geoffroy Saint-Hilaire's brazen assertion that, "There is philosophically speaking only a single animal." Broca's fellow countrymen, Achille Foville, with his concentric ring model of the hemisphere; and Pierre Gerdy's annular circonvolution, look backward to Geoffroy, and presage Broca's great limbic lobe proposal. In the examination of limbic lobe description by $19^{\text {th }}$ century apatomists, one is presented with some of the early and more alluring large scale geometric models put forth to explain and unify mammalian hemisphere architecture.

The Development of Our Conceptual Understanding in Neuroanatomy: Placing Broca's Great Limbic Lobe in a Continuum of Ideas Dating From the Early Nineteenth Century to the Present Day:

Examining both the early $19^{\text {th }}$ century origins of Broca's proposal on the limbic brain, and the relationship of Broca's construct to ideas on hemispheric anatomy which came after his proposal is an important exercise which, if followed, can help shed light on the development of our concepts of hemispheric neuroanatomy. The following brief examples trace developments in limbic lobe anatomy after Broca demonstrated the fruitfulness of this exercise. Broca put forth his proposal twenty years before the advent of "modern" cytoarchitectonic description (remember that Broca was working at a relatively early age in the science of microscopy). With the maturation of cytoarchitectonics in the closing decades of the $19^{\text {th }}$ century, it would be seen that the hemispheres evolutionary old cortex is contained in Broca's idea of the limbic lobe (or equivalently Broca's lobe would be seen as compromising the hemisphere allo-and periallocortical investments (9, p. 259)). The first characterization of Broca's lobe employing recognizable modern cytoarchitectonic lexicon may date to E. A. Schaefer's TextBook of Physiology (1900); More recent ideas in the field of comparative 
hemispheric topology can also be employed to recast Broca's proposal. Incorporating these ideas with their attendant terminology reveal Broca's lobe to be a derivative of the medial pallium and neighboring sectors of the dorsal and lateral pallium.

The Uniqueness of the Temporal Lobe and Limbic System: A point made most tellingly in Gloor's recent text, is that the temporal lobe and its limbic components particularly in higher primates represents a macrostructure for a privileged interaction of isocortical, allocortical, and subcortical (amygdala) neural structures. Such an anatomic interrelationship is not seen to the same degree in less temporalized forms. The conjunction of visual input (inferior temporal lobe) and auditory input (superior temporal lobe) at the hilar limbic threshold compels one to reflect on the behavioral implications of this unique anatomy. Gloor comments: "This evolutionary development probably has profound implications for the way we as humans experience and interact with the world around us." $(15, \mathrm{p} .12)$. An examination of the temporal lobe and its limbic infrastructure would appear motivated in part by the very structure itself.

\section{REFERENCES}

1) MacLean, P. (1952). Some psychiatric implications of physiological studies on frontotemporal portion of limbic system (visceral brain), Electroencephalography: The basal and temporal regions, Yale J. Biol. Med. 22, 407-418.

2) Swanson, L. W. (1987). Chapter Entitled, Limbic System, in Encyclopedia of Neuroscience (G. Adelman, ed.), Birkhauser.

3) Broca, P. (1878). Anatomie comparee des circonvolutions cerebrales. Le grand lobe limbique et la scissure limbique dans la serie des mammiferes, Rev. Anthropol. 1, Ser, 2, 385-498.

4) Ramon y Cajal, S. (1996). Recollections of My Life. MIT Press.

5) Ariens Kappers, C. U., Huber, G. C., and Crosby, E. C. (1936/1967). The Comparative Anatomy of the Nervous System of Vertebrates, Including Man. Hafner Publishing Company, New York.

6) Nauta, \#: J. H., and Fiertag, M. (1986). Fundamental Neuroanatomy, W. H. Freeman xT Company, New York.

7) Herrick, C. J. (1933). The functions of the olfactory parts of the cerebral cortex, Proceeding of the National Academy of Sciences, pp. 7-14.

8) Papez, J. (1937). A proposed mechanism of emotion, Arch. Neurol. Psychiatry, 38, 725-743.

9) MacLean, P. (1990). The Triune Brain in Evolution: Role in Paleocerebral Functions, Plenum Publishing.

10) Yakovlev, P. I. (1948). Motility, behavior, and the brain. Stereodynamic organization and neural coordinates of behavior, J. Nerv. Ment Dis. 107, pp. 313-335.

11) Olds, J., and Milner, P. (1954). Positive reinforcement produced by electrical stimulation of septal areas and other regions of the rat brain. J. Comp. Physiol. Psychol. 47,419-427. 
12) Heimer, L., and Wilson, R. D. (1975). The Subcortical Projections of the Allocortex: Similarities in the Neural Associations of the Hippocampus, the Piriform Cortex, and the Neocortex, in: Golgi Centennial Symposium. Proceedings (M. Santini, ed.), Raven Press, New York, pp. 177-193.

13) Mogenson, G. J., Jones, D. L., and Yim, C. Y. (1980). From motivation to action: Functional interface between the limbic system and the motor system, Progress in Neurobiology, pp. 69-91.

14) Northcutt, R. G. (1969). Discussion of the preceding paper. Annals New York Academy Sciences, 167: 180-185.

15) Gloor, P. (1997). The Temporal Lobe and Limbic System, Oxford University Press. 